\title{
Síndrome de Donohue (leprechaunismo)
}

\author{
Dr. Carlos Toro A. ${ }^{\text {; }}$ Dra. Francisca Ugarte P. ${ }^{2}$; Dr. José Ignacio Infante L. ${ }^{3}$; \\ Dr. Samuel Benveniste I .4 ; T.M. Julia Cavada P. 4
}

\section{Donohue's syndrome}

A five month old girl with phenotypic features of Donohue's syndrome, is presented. The diagnosis was confirmed by the presence at the pathologic examination of cystic degeneration of the Hassall's corpuscles of the thymus, increased iron deposits at the liver, hyperplasia of the Langerhans islets and predominance of Beta cells at the pancreas. A literature review wals undertaken and some of the pathogenic mechanism involved are discussed.

(Key words: Eeprechaunism, Donohue's syndrome, histologic tindings, liver, pancreas, $\beta$ cell Langerhans islets hipoglycemia, marasmus).

Donohue describió en 1948 un niños que tenía una facie peculiar $y$ un conjunto de altera. ciones fenotípicas, lo que denominó "disendocrinia"l. En 1954 con lá publicación de dos casos nuevos ${ }^{2}$ se cambió el nombre de este síndrome por el de "leprechaunismo", ya que las características físicas de estos pacientes recordaban los gnomos del folklore irlandés.

En li literatura se han descrito numerosos casos de este síndrome y se ha planteado una probable herencia autosómica recesiva ${ }^{3}$. Su patogenia estaria relacionada a una resistencia celular a los efectos metabólicos de la insulina, factor de crecimiento epidérmico y somatomedina $\mathrm{C}^{4,5,6}$

El propósito de esta presentación es describir un niño con características fenotípicas del síndrome y su confirmación anatomopatológieu, lo que no hemos encontrado en la literatura nacional.

\section{Caso clínico}

Niña de 5 meses, producto de un embarazo de término que habría cursado con polihidroamnios, nacida mediante cesárea por ruptura prematura de membranas, con peso de nacimiento de

1. Departamento de Pediatría y Cirugía Infantil Central, Facultad de Medicina, Universidad de Chile.

2. Becada de Pediatría, Departamento de Pediatría y Cirugía Infantil Sur, Facutlad de Medicina, Univer sidad de Chile.

3. Departamento de Pediatría y Cirugía Infantjl Sur, Facul tad de Medicina, Universidad de Chile.

4. Servicio de Anatomía Patológica, Hospital Exequiel González Cortez, S.M.S.S.
$2970 \mathrm{~g}$, talla $47 \mathrm{~cm}$ y Apgar 8 al primer y quinto minuto.

Hospitalizada 2 meses 18 días en neonatología, donde presentó 5 episodios caracterizados por apatia, rechazo alimentário con y sin vómitos, dificultad respiratoria de grado variable $y$ aumento del número y contenido líquido de las deposiciones. Todos estos episodios habrían cedido coincidiendo con el reemplazo de fórmu. las lácteas "maternizadas" por leche sin lactosa y la administración simultánea de solución de glucosa $5 \%$ endovenosa.

Ingresó al servicio de pediatría a los 2 meses 19 dias de edad, pesando $2.480 \mathrm{~g}$, con una talla de $49 \mathrm{~cm}$, circunferencia craneana y torácica de 36 y $29 \mathrm{~cm}$ respectivamente, facie de gnomo, pies y manos grandes, piel apergaminada, panículo adipose muy disminuido, bola de Bichat conservada; frente alta y cuadrada, fontanela anterior de $5 \times 3,5 \mathrm{~cm}$ y posterior de $2 \times 1,5 \mathrm{~cm}$ (plano sagital y fronta); sutura frontal abierta $y$ sagital amplia; macroftalmía e hipertelorismo; orejas alargadas, de lóbulo prominente e implantación baja; micrognatia y paladar ojival; escoliosis de convexidad dorsal a izquierda y lumbar a derecha; soplo sistólico 2/6 en tercer espacio paraesternal izquierdo no irradiado. No se encontró visceromegalia ni alteraciones genitales.

Evolucionó con deterioro progresivo del peso, a pesar de aportes habituales de 150 a $200 \mathrm{Cal} x$ $\mathrm{kg} x$ dia $y$ nunca inferiores a $90 \mathrm{Cal} \times \mathrm{kg} \times$ dia. Durante su hospitalización presentó tres episodios similares a los descritos en neonatología, agregándose en los dos últimos crisis de apnea con bradicardia, hipotermia y lentitud circulatoria periférica. Todos fueron tratados con hidratación parenteral, prevención de úlceras de 
situaciones de apremio y antibioterapia (los cultivos fueron repetidamente negativos). En el último episodio se comprobaron glicemias de 30 y $34 \mathrm{mg} \%$, a pesar de haber estado recibiendo soluciones de glucosa al $5 \%$ y $10 \%$ respectivamente por vía endovenosa. La paciente falleció a los 74 días de hospitalizaciótı.

Los exámenes de laboratorio dieron los siguientes resultados: sedimento de orina, natremia, kalemia, calcemia y fosfatasas alcalinas normales. Ilemograma con desviación izquierda; proteinemia de $5,8 \mathrm{~g} \%$, albumina de $3,42 \mathrm{~g} \%$. globulina de $2,38 \mathrm{~g} \%$, Alfa 1 de $0,22 \mathrm{~g} \%$ $(n=0,13$ a 0,4$)$, Alfa 2 de $0,8 \mathrm{~g} \%(n=0,44$ a $1,1)$, Beta 1 de $0,41 \mathrm{~g} \%(\mathrm{n}=0,3$ a 0,6$)$, Beta $2 \mathrm{de}$ $0,18 \mathrm{~g} \%(\mathrm{n}=0.2$ a 0,74$)$ y Gamma de $0.77 \mathrm{~g} \%$ ( $n=0,67$ a 1,41$)$. Aminoaciduria negativa $y$ aminoácidos séricos sin alteraciones. TSH 0,2 $\mu \mathrm{UI} \times \mathrm{ml}(=0.5$ a 5$\left.), \mathrm{T}_{4} 2 \mu \mathrm{g} \times \mathrm{d}\right](\mathrm{n}=5$ a 12$)$ y $\mathrm{T}_{3} 56 \mathrm{ng} \times \mathrm{ml}(\mathrm{n}=50$ a 200). Reacción de Fehling en deposiciones negativas. Electromiografía y ecografía de cráneo nomal; radiografía de tórax: silueta cardiovascular normal y enfisema pulmonar.

En el examen de anatomia patológica ademas de los hallazgos físicos descritos, se encontró disminución generalizada del peso y tamaño viceral, disminución importante del tamaño del timo y sangre en el lumen gástrico. Microscópicàmente se encontró congestión y edema encefálico, transformación quistica de los corpúsculos de Hassal y congestión del timo (Fig. 1). El hígado estaba congestivo y con abundante piz:mento de ficro en el citoplasma de lus hepatocitus (Figs. ? y 3). En el páncreas había hiperplasia de los islotes de Langerhans y aumento del número de células Beta en relación a células $A$ lfa $(F i g .4)$; evidencia de infección pulmonar terminal.

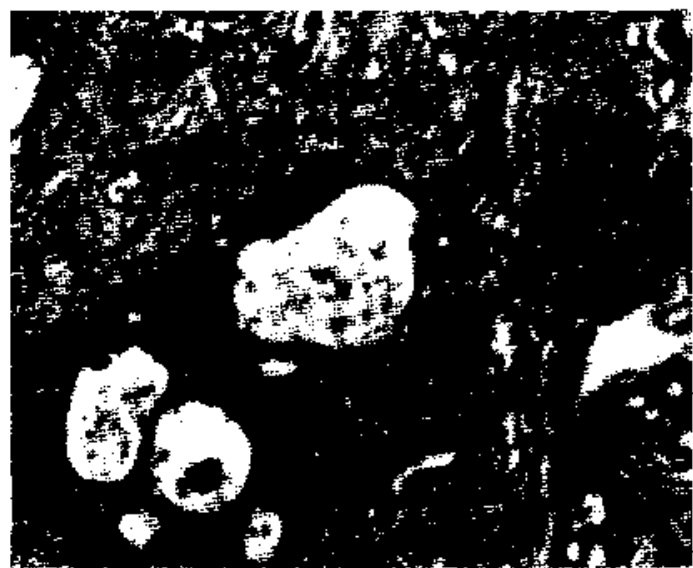

Figura I. Timo: congestión tisular y degeneración quística de los corpúsculos de Hassull. Tinción: hematoxilina cosina. Anmento: $60 \mathrm{x}$

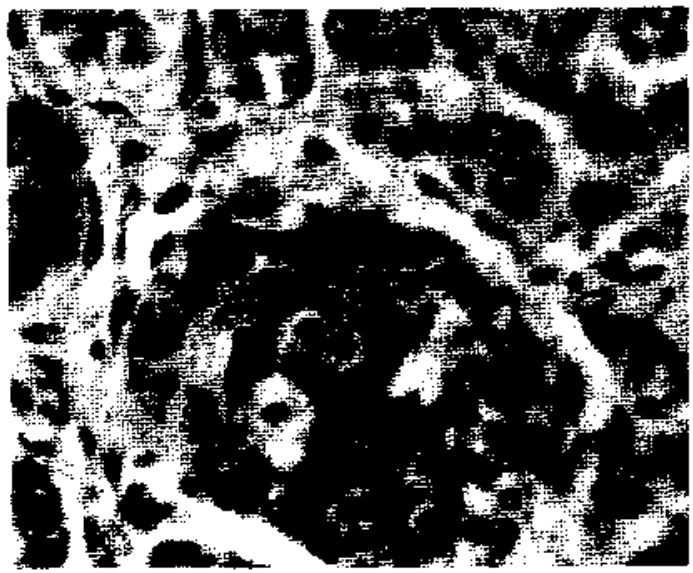

Figurn 4. Páncteas: hiperplasia de los islotes do !angerhans y predominio de células Beta sobre Alfu. Tinctón: de aldehido fucsina para células Beta dè páncreds) Aumento: $400 \mathrm{x}$
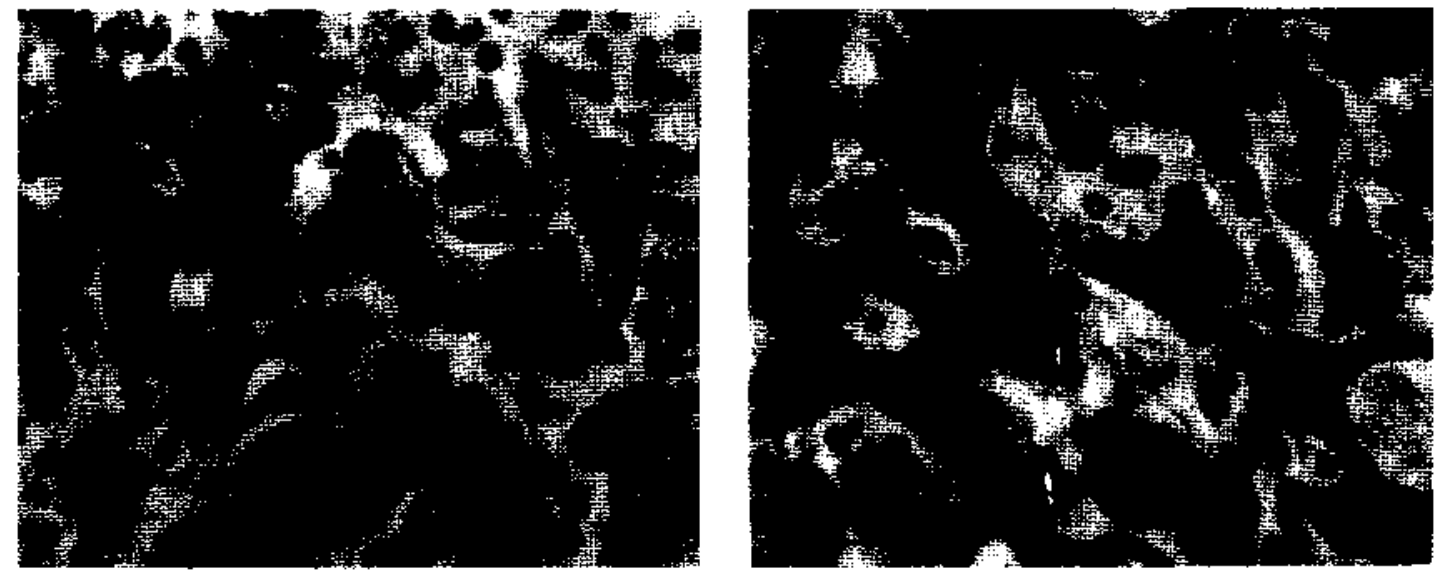

Figura 2 y 3: Hígado: Congestión tisular. Abundante pigmento de fierto en el citoplasma de los hepatocitos. Tinción de Mallory modificada para fierro. Aumento: 160 x y $400 \mathrm{x}$ respectivamente. 


\section{DISCUSION}

Nuestro paciente presentó desđe su nacimiento algunos rasgos físicos caracteristicos del sindrome de Donohue $3,2,9$, que permitieron sospechar el diagnóstico. Además presentaba otros que, si bien son menos frecuentes, también han sido descritos en estos pacientes: paladar ojival, aumento del tamaño de las fontanelas, soplo sistólico y antecedente de polihidroamnios en el embarazo $11,10,12,7$. Además tenía escoliosis, que no ha sido descrita anteriormente y proba. blemente se trate de una malformación asociada independiente del síndrome de base.

Su evolución con marasmo precoz, se ha visto en todos los casos previamente descritos y la muerte temprana sucede en $64 \%$ de los pacien. tes 10 .

A los hallazgos clínicos descritos, agrega la confirmación de laboratorio de hipoglicemia $^{8,9.10}$, que en estos pacientes no son permanentes sino desencadenadas en relación con situaciones de ayuno moderado ${ }^{7}$. En lo que respecta a la hipoproteinemia encontrada en esta niña ${ }^{12}$, cuya explicación como parte del síndrome no está clara aún, existen evidencias experimentales, en cultivos de fibroblastos de una relación entre la resistencia celular a la insulina con niveles bajos de sintesis y altos de degradación de proteínas ${ }^{6,4}$

Además, Jos hallazgos anatomo-patológicos (bajo peso de todos los órganos ${ }^{11}$, ausencia dc panículo adiposo ${ }^{8,11}$, transformación quistica de los corpúsculos de Ilassall ${ }^{11}$, depósitos de fierro en el higado $1,2,11$, hiperplasia de los islotes de Langerhans y aumento de células Beta del páncreas) $)^{1}, 3,8,10,11$, confirman el diag. nóstico. Siendo dichas alteraciones estructurales de las más características del síndrome de Donohue, es recomendable buscarlas dirigidamente en los pacientes con marasmo maligno $y$ rasgos fenot ípicos compatibles.

\section{RESUMEN}

Se presenta el caso de una niña de 5 meses, con un fenotipo compatible con sindrome de
Donohue; confirmándose el diagnóstico en la anatomia patológica por la presencia de transformación quística de los corpúsculos de Hassall en el timo, abundante pigmento de fierro en el hígado e hiperplasia de los islotes de Langerhans y aumento de células Beta en el páncreas. Se revisa la literatura y se discuten los posibles mecanisnos patogénicos involucrados.

\section{REFERENCIAS}

1. Donohue, w.L.: Dysendocrinism. J Pediatr 32: 739 , 1948.

2. Donohue, W.I., Uchica, I.: Leprechaunism, a euphuism for a rare familial disorder. J Pediatr 45 : 505,1954

3. Der Kaloustian, V.M, M.D., Kronfol, N.M., M.D., Takla, R., M.D., Hahash, A., Khazin, A., M.D., Najor, S.S., M.O.: Leprechaunism. Am J Dis Child 122: $442,1971$.

4. Kaplowitz, P.B., and D'ercole, A.J.: Fibroblast from a patien with leprechaunism are resistant to insulin, epidermal growth factor and somatomedin C J Clin Endocrinol Metab 55: 741, 1982

5. Frindik, J.P., M.D., Kemp, S., Ph.D., Fiser, R., M.D., Schedewie, M., M.D., Etders, M.J., M.D.: Phenotypic expression in Donohue syndrome (Leprechaunism): A role for epiderml growth factor. I Pediatr 107: 428, 1985.

6. Ballerd, F.J., Read, L.C. and Gunn, J.M.: Protein synthesis and breakdown rates associates with the insulin resistence of fibroblast from patients with leprechaunism, I Clin Endocrinol Yetab 61: 1146, 1985 .

7. Dekaban, A.: Metabolit and chromosomal studies in leprechaunism. Arch Dis Child 40: 632, 1965.

8. Rotn, S., M.D., Schedewie, H., M.D., Herzberg, V., Ph.D., Olefsky, J, M.D., Elders, M.J., M.D., Rubenstein, A., M.D.: Cutaneous manifestations of leprechaunism. Arch Dermatol 117: 531, 1981.

9. Bergsma, D.: Birth defect compendium, 2nd Fd. National Foundation. Marchof Dimes, New York $1979,644$.

10. Rosenberg, A.M. M.D., Haworth, J.C., M.D., Degroot, G.W., M.D., Trevenen, C.L., M.D., Rechler, M.M., M.D.: A case of leprechaunism with severe hyperinsulinemia. Am J Dis Child 134: 170, 1980.

11. Källó, A., Lakatos, I. and Saijairtó, L.: Leprechaunism Donohue's syndrome). I Pediatr 66: 372 1965.

12. Gross-Kieselstein, E., M.D., Ben-Galim, E., M.D., Amir, N., M.D., Abrahamov, A., M.D.: Leprechaunism (Donohue syndrome), Amer J Dis Child 126: $500,1973$. 